\title{
Belphégor
}

Littérature populaire et culture médiatique

17 | 2019

Mutations des légitimités dans les productions

culturelles contemporaines

\section{Dossier « Mutations des légitimités dans les productions culturelles contemporaines »}

Anne Besson, Anne Isabelle François, Sarah Lécossais, Matthieu Letourneux and Anne-Gaëlle Weber

\section{(2) OpenEdition}

\section{Journals}

Electronic version

URL: https://journals.openedition.org/belphegor/1923

DOI: 10.4000/belphegor.1923

ISSN: 1499-7185

Publisher

LPCM

Electronic reference

Anne Besson, Anne Isabelle François, Sarah Lécossais, Matthieu Letourneux and Anne-Gaëlle Weber, "Dossier « Mutations des légitimités dans les productions culturelles contemporaines »", Belphégor [Online], 17 | 2019, Online since 08 May 2019, connection on 25 August 2021. URL: http:// journals.openedition.org/belphegor/1923 ; DOI: https://doi.org/10.4000/belphegor.1923

This text was automatically generated on 25 August 2021.

\section{(i) $\$(\Theta$

Belphégor est mis à disposition selon les termes de la Licence Creative Commons Attribution - Pas d'Utilisation Commerciale - Pas de Modification 4.0 International. 


\title{
Dossier « Mutations des légitimités dans les productions culturelles contemporaines »
}

\author{
Anne Besson, Anne Isabelle François, Sarah Lécossais, Matthieu \\ Letourneux and Anne-Gaëlle Weber
}

Introduction

On sait combien le contexte culturel de la postmodernité apparaît marqué par une déhiérarchisation des pratiques «savantes " et "populaires » de plus en plus nette, accentuée encore par l'usage des outils numériques.Les réflexions réunies ici entendentinterroger les conséquences très actuelles d'une telle hybridation (supposée ou non) des composantes de la "culture » sur la recherche et l'enseignement de la littérature et sur la définition de son objet, en concentrant précisément son attention sur la manière dont les processus de « légitimation » de la "culture » et des « savoirs » ont pu changer et évoluer, voire s'opposer ou se confondre avec la "vulgarisation» savante. L'accès du plus grandnombre à un certain type de recherche universitaire ou encore à des procédés d'écriture ou de commentaires entraîne-t-il nécessairement, en retour, une redéfinition non seulement du savoir académique en littérature, mais aussi de ses objets et de ses catégories? Pour mesurer ou cerner ces éventuels «bouleversements» très contemporains, on verra que s'impose régulièrement le détour vers l'histoire des processus croisés de légitimation et de vulgarisation ${ }^{1}$.

2 Le dossier décline trois grandes problématiques, découlant des articulations possibles entre les termes de " savoirs ", " culture ", " populaire » et " légitime ", dont il s'agit à chaque fois d'interroger les sens ${ }^{2}$.

\section{Mutations de l'expertise critique}

3 On s'intéressera dans cette partie auxmouvementssymétriques de légitimation et de diffusion populaire ou de vulgarisation, à l'histoire des processus à l'œuvre dans la légitimation des savoirs populaires et symétriquement des pratiques lectoriales et de la 
critique érudite, enfin aux relations entre «érudition » et " constitution des savoirs académiques ».

Le champ de l'expertise critique, entend-on souvent ces dernières années, serait en crise ; du moins subirait-il une profonde mutation, qui conduirait d'une traditionnelle verticalité (le savoir se diffusant de haut en bas, du "sachant " au néophyte) à une nouvelle horizontalité, celle des pairs connectés, des communautés d'amateurs partageant leur intelligence collective, via les réseaux, sociaux en particulier. Il convient donc de s'intéresser auxformes des savoirs produits par les « amateurs » (par ceux qui, au sens large, ne relèvent pas d'institutions académiques), que ce soit autour de découvertes savantes ou de productions artistiques, très souvent conçues comme "populaires ", et d'observer la manière dont ces savoirs se distinguent ou non de la production académique pour faire émerger un type d'érudition spécifique. On y trouvera sans doute matière à nuancer quelques partis-pris attachés autant à la critique des amateurs (subjectivisme, expression de l'émotivité, point de vue «immergé »...) qu'aux oppositions entre savoirs académiques et savoirs populaires.

5 De telles tensions ne sont en effet pasnouvelles, comme nous le rappelle l'article d'Anne-Gaëlle Weber qui fait le point sur les définitions fluctuantes et les évaluations ambivalentes qui ont été données au XIXede la notion d'« érudition », opposée ou non $\mathrm{au}$ " bon » savoir et au savoir académique, et de la figure de l'érudit - souvent moqué en provincial maniaque du détail. Rémy Arcemisbéhère et Juliette Mascart nous montrent à l'inverse, à partir du cas de deux auteurs à la posture spécifique dans le champ littéraire du début du XIX ${ }^{e}$ siècle, Nerval et Stendhal, comment des artistes investissent le domaine du savoir et de la critique en revendiquant la singularité « dilettante » de leur point de vue.

6 L'érudition spécialisée sur les objets populaires a d'abord concerné de petites communautés aux membres fortement investis développant l'usage de formes ou de protocoles tout à la fois savants et ludiques. C'est le cas par exemple des «bédéphiles » de la première heure (leClub des Bandes Dessinées et son bulletinGiff Wiff ${ }^{3}$ ), ou bien des amateurs du plus fameux personnage créé par Maurice Leblanc, Arsène Lupin - l'article de Cédric Hannedouche se consacre à faire connaitre les publications amatrices qui lui ont été consacrées par les communautés de passionnés, depuisLa Gazette des Études Lupiniennesà partir de 1965 jusqu'àL'Aiguille Preuve, où ce même Cédric Hannedouche publie aujourd'hui une partiede ses recherches ${ }^{4}$.La prégnance d'Internet sur nos vies culturelles aujourd'hui a cependant permisun transfert d'influence décisif des critiques érudits, savants professionnels, et seuls aptes à conférer la légitimité, vers un pouvoir aux consommateurs, lecteurs, joueurs, spectateurs, fans. Au passage, de nouveaux équilibres s'instaurent, donnant plusde visibilité à des domaines traditionnellement mal-aimés du champ culturel, séries, genres de l'imaginaire et œuvres "transgénérationnelles " (les romans populaires du XIX siècle, les fascicules bon marché, les magazinespulp, les journaux de bandes dessinées ou aujourd'hui les productions des médias de flux, telles que les soap opera, séries ou jeux en ligne), et à ceux qui s'y intéressent et souhaitent rendre publique leur passion.

7 L'accès facilité aux moyens de mener et de diffuser le savoir via Internet a bouleversé ces modalités de partage. Les forums par exemple permettent le développement de débats critiques, aussi érudits qu'éruptifs, comme celui, fameux dans le milieu concerné, consacré à la métaphysique en science-fiction, sur lequel reviennentpour nous Irène Langlet et Simon Bréan : en tant qu'observatrice et que participant, ils 
montrent à la fois combien des positions théoriques peuvent émerger de ce type d'échanges et être débattues, et combien cette production réflexive nourrie reste cependant étrangère aux enjeux de stratification commune du savoir scientifique. Abordant un autre exemple de conversation entre amateurs "pointus", Bounthavy Suvilay se penche sur les discussions entre lecteurs de manga, qui vont chercher les toutes dernières parutions sur des sites non légaux, ce qui témoigne d'un suivi exigeant et d'un investissement passionné; elle propose une analyse descommentaires qu'ils échangent, de manière asynchrone et mondialisée, via l'interface Disqus (qui intègre des fonctionnalités propres aux réseaux socionumériques), et dégage une typologie des savoirs spécialisés qui s'y donnent à lire.

On ne peut que noter l'explosion des contenus justifiant un tel déploiement d'érudition, et cela n'a sans doute rien d'une coöncidence. Lemultivers connecté des superhéros de comics n'a jamais été si exploité, les mondes secondaires comme ceux de Tolkien ou à plus forte raison de George Martin (encore en cours de déploiement, certains de ses segments sont énigmatiques et aimantent donc l'activité herméneutique), les séries télévisées qui demandent à leur public un investissement majeur : tous ces produits font fonds sur une même expertise toujours plus raffinée de leur public. Xavier Lorenzo et Sylvie Périneau-Lorenzo choisissent justement l'exemple deGame of Thronespour observer ce qu'ils conçoivent comme une véritable «reconfiguration du processus d'expertise » par la praxis amatrice dans la sphère privée; ils invitent ainsi les institutions scolaires à prendre en compte les compétencesmultiples déployées dans la production de fanvidéos sur Youtube.

9 Bouclant la boucle, la mise à disposition instantanée de masses de connaissances placées sur le même plan, potentiellement par chacun, pour chacun, conduit la communauté universitaire en sciences humaines à de profonds remaniements de ses pratiques et de ses hiérarchies. L'émergence descultural studies, puis desfan studieset de la figure de l'«aca-fan » (posture de recherche visant l'hybridation entre «academic » et «fan») constitue undes signes de cette évolution qui intéressedirectement nos réflexions sur la construction de la légitimité culturelle : lesfan studies, théorisées il y a plus de 15 ans par Henry Jenkins aux États-Unis, se sont plus récemment imposées en France, et il s'agit aussi de faire le point sur ces nouveaux usages, avec l'article de Mélanie Bourdaa qui ouvre un chantier méthodologique interrogeant la position d'« ethno-fan » et la possibilité d'unecyberethnographie participative pour l'étude des communautés de fans.

\section{Histoire de la légitimation et effets d'hybridation au sein des œuvres}

On assiste depuis plusieurs décennies à une accélération des effets d'hybridations entre des pratiques qui semblaient appartenir auparavant à des sphères culturelles hétérogènes. Ilconvient donc d'examiner, dans une perspective à la fois synchronique et diachronique, la manière dont des œuvres dites "légitimes" empruntent aux cultures populaires et, à l'inverse, la manière dont des œuvres dites "populaires » empruntent aux savoirs et aux cultures légitimes.

Le pouvoir "distinctif» d'une pratique culturelle, tout comme le "répertoire de goûts » des individus (pour utiliser les lexiques respectifs de Pierre Bourdieu et Richard 
Peterson ${ }^{5}$ ) ne se comprennent que dans un contexte social donné ; ils sont le fruit d'une histoire, que leurs bouleversements actuels dans le sens d'une difficulté de plusen plus grande à maintenir les distinctions entre " populaire » et "savant ", " bas » et " haut ", commande d'interroger. Le cas de la bande dessinée en France est particulièrement parlant, puisque la façon dont elle est perçue a déjà suffisamment muté pour faire l'objet d'un ample retour réflexif : l'article de Florian Moine propose ainsi l'histoire de son institutionnalisation - des questionnements qu'elle a soulevés, des limites à laquelle elle se heurte - à travers celle du Musée de la Bande Dessinée d'Angoulême, tandis que celui de Sylvain Lesage porte sur une étape critique essentielle, l'affirmation du «passage à l'âge adulte »de la bande dessinée dans l'article séminal deLuc Boltanski, «La constitution du champ de la bande dessinée », paru en 1975 - ou comment un gain en terme de légitimation peut néanmoins signifier l'occultation de pans entiers de la production et des usages en amont et en aval.

Si des travaux ont été menés sur la constitution dans l'histoire de panthéons littéraires et scientifiques ou sur "l'invention des classiques ${ }^{6}$, il convient de poursuivre l'enquête sur l'historicité des critères de valeur - et de dévalorisation. On peut considérer en effet les distinctions et leur pouvoir classant comme un produit de la modernité, notamment littéraire, et le fruit de stratégies au sein des mondes de l'art dont le postmodernisme (et le " post-post » contemporain) se seraient saisis, pour les subvertir et/ou les consolider. Mais la montée en force des cultures mass-médiatiques d'une part, et des pratiques participatives d'autre part, fragilise les modèles distinctifs sur lesquels reposait l'idée de légitimité, dont l'unité est mise à mal notamment par les rapports de forces entre classes, genres, origines ethniques et géographiques, identités sexuelles, dont rendentcompte les réflexions initiées parles cultural studies. Ne faut-il pas dès lors détacher les logiques de distinction et les pratiques de légitimation de la perspective d'une valeur ou d'une légitimité objectivées? La question est posée dans l'article deThomas Mercier-Bellevue qui s'interroge sur lediscrédit dont souffrirait la "popmainstream» dansla critique d'art, étant entendu que celle-ci «ne prend pas seulement acte de légitimités existant en-deçà d'elle, mais qu'elle est aussi et surtout un opérateur de transformation ontologique, un opérateur d'artification ». Il explique cette délégitimation notamment par l'importance du paradigme moderniste de la "traductibilité herméneutique », pour lequel l'œuvre d'art ne doit pas être d'emblée lisible mais se révéler par une opération d'interprétation.

Il faut également tenir compte de la fragmentation des instances légitimantes et de la montée en puissance de systèmes rivaux de légitimation (médiatiques, sous-culturels, contre-culturels), si bien que laquestion d'une légitimité objectivée laisse de plus en plus la place à des légitimations concurrentes, ce qui en altère profondément les principes. L'article de Sylvie Ducas sur l'histoire des prix littéraires français, qui se sont multipliés de manière exponentielle plus d'un siècle après la création du Goncourt, témoigne bien d'une telle évolution en trois temps : «l'autorité institutionnelle et ses crises, la déterritorialisation du goût et de la valeur, et enfin l'atomisation d'une légitimité à la carte» marquée par "l'intériorisation des logiques marchandes inhérentes aux industries culturelles » - ce qui n'empêche nullement le système des prix, avec leur gain symbolique et économique, de fonctionner parfaitement bien !

Peut-être conviendrait-il alors de penser desdegrésde légitimation, sur le modèle historiquement illustré par la catégorie du middlebrow,cettemasse de textes composant la littérature « moyenne », majoritaire et pourtant peu affectée, ou peu abordée, par les 
débats critiques commepar les stratégies de légitimation qui ont accompagné l'esthétisation et l'artification de pratiques et de biens culturels dans leurs formes les plus marchandes et les plus prosaïques. L'article de Diana Holmes, qui signe un ouvrage récent sur la question ${ }^{7}$, revient aux sources d'une «illégitimité » liée à la féminisation de ce champ, et lit sa possible réévaluation dans les évolutions d'une critique qui tend à réhabiliter la lecture de plaisir, immersive et affective.

\section{Nouvelles formes et nouvelles pratiques}

Les transformations des pratiques de la vulgarisation et des écritures critiques chez les amateurs et lessavants, leur manière d'explorer des formes d'expression originales rendues possibles par les nouveaux médias, invitent les disciplines universitaires à un retour réflexif sur leurs propres méthodes et leurs objets, leur manière de construire les savoirs et les moyens de diffusion qu'elles privilégient.

Nous assistons en effet à une explosion des supports de vulgarisation scientifique : blogs, vidéos, bande-dessinées, romans, expositions, conférences, essais, ateliers, théâtre, illustrations, etc. Le développement récent, dans l'université française, de nouveaux modes d'enseignement, sous la forme des MOOC, qui ouvrent lesrecherches en lettres à un nouveau public, propose ainsi de nouvelles formes de diffusion et, dans le même temps, redéfinit peut-être ledomaine même de la recherche scientifique ainsi que celui de l'enseignement académique. Suivant une approche politique, l'émergence du concept de « citizen science » (science citoyenne) et la (re)politisation du champ du savoir ${ }^{8}$ soulignent la mobilisation des connaissances de la société civile en vue d'une coconstruction des savoirs, ou afin d'orienter les recherchesvers l'intérêt commun, pour élaborer un nouveau régime de savoir. Le lien entre choix scientifiques ou technologiques et choix politiques étant reconnu, le modèle de participation du public porte en lui un potentiel qui pourrait profondément changer la nature du travail académique, le rôle des citoyens et le jeu démocratique. Comment s'opère cette aptitude à constituer et transmettre des savoirs critiques, autrement dit des savoirs conscients de leurs mécanismes d'élaboration, de leurs enjeux et deleur valeur dans le champ social? Quelles formes prennent ces pratiques entendant exploiter la dimension opératoire des humanités, contribuant à mettre en question les partages établis et l'économie des savoirs? Comment penser cette approche et quelles en sont les incidences sur les frontières entre science et opinion et les dialogues entre savoir et pouvoir?

17 Il est donc nécessaire, pour la recherche en littérature et sciences humaines, de réfléchir aux moyens de sa propre diffusion, d'examiner les (nouvelles) formes d'écriture entendant transmettre le savoir au grand public, et de montrer combien la diffusion permise par les outils numériques a pu modifier le sens même du "savant ", du «populaire » et les procédés de légitimation: l'usage des MOOC n'est-il pas un moyen de vulgarisation participant de la légitimation des savoirs? C'est dans cette perspective que s'inscrit l'article de Marie-Lucie Bougon, qui y rend compte des premiers résultats de sa vacation dans le cadre du projet LégiPop : elle a travaillé sur l'exploitation des données présentes sur les forums durant les trois sessions duMOOC fantasy créé par l'université d'Artois sur la plateforme FUN (France Université Numérique), et nous présente son étude des dynamiques d'apprentissage mises enplace entre lesapprenants eux-mêmes. 
18 L'invention de ces nouvelles formes et des nouveaux espaces de médiatisation - la "Petite bédéthèque des savoirs » lancée par les Éditions Lombard, les chaînes de youtubeurs pour apprendre l'histoire antique ou la mythologie grecque, etc. - sontelles des exemples et leviers permettant l'émergence de véritables contre-propositions médiatiques de la part des SHS ? La mise en publicité des savoirs et des résultats de la recherche, leur circulation sur des supports variés entre monde universitaire et société civile, largement traitée dans le cas des sciences dites dures ${ }^{9}$, demeure encore un impensé dans le cas deshumanités, des SHS et des étudeslittéraires. L'écriture du savoir est soumise à des règles et des contraintes pérennisées à travers des textes et des objets, relais d'une représentation d'un monde, dépendant du contexte culturel, économique et politique dans lequel ils se réalisent, mais aussi en constante mutation, suivant les mouvements d'évolution des savoirs eux-mêmes. Le développement d'une pratique comme les "carnets de thèse ", hébergés en France sur la plate-forme de référence du savoir scientifique enopen accessHypotheses.org, en constitue un exemple particulièrement flagrant, auquel est consacré l'article de Mélodie Faury. Celle-ci propose une typologie des billets proposés au lectorat par ces blogs d'un nouveau type, donnant à voir un apprentissage de la recherche en train de se faire, une réflexivité au plus près du quotidien du jeune chercheur.

Le savoir académique pourrait ainsi se dire sous d'autres formes qui n'apparaissentpas toujours comme du «savoir » ou une écriture «savante » : il peut prendre des formes non universitaires, inventer des écritures de vulgarisation par la remédiation de discours savants (BD, twitter) ou par l'investissement des supports numériques. Lavulgarisation ou la "popularisation » (pour reprendre le terme en cours en France au XVIII ${ }^{\mathrm{e}}$ siècle dont l'équivalent anglais « popularization » n'est pas chargé de connotations péjoratives) s'oppose-t-elle encore à la «légitimation »? L'une et l'autredémarches semblent au contraire tendre à se confondre dans l'usage ou l'échange de formes communes. A cet égard, l'essai constitue un terrain d'étude privilégié pour penser ces questions des frontières du savant et du rôle critique des humanités à traversune médiatisation susceptible de toucher la sphère extra-académique : l'article de Maxime Hureau soulève ces problématiques en se concentrant sur un exemple d'essai en bande dessinée,Le Déploiementde Nick Sousanis(qui a choisi de publier sa thèse sous ce format). Maxime Hureau prend ses distances avec cette démarche, malgré le succès rencontré, en en pointant aussi les limites épistémologiques et les impensés politiques.

Enfin, notre démarche de recherche, réflexive et ouverte, ne serait pas en accord avec elle-même si elle ne consacrait pas une part de ses ressources à mettre à son tour à disposition du public le savoir académique produit à propos de ses propres pratiques savantes. Nous renvoyons donc pour finir cette présentation des résultats (provisoires) de nos travaux à l'opération de valorisation auxquels ils ont donné lieu : l'« exposition en ligne " LégiPop, "L'érudition des amateurs », site richement illustré, propose une exploration de l'histoire, des questionnements et des productions du savoir fanique à travers les grands univers de la pop culture, de Tolkien à Rowling.

Bibliographie : ouvrages critiques de référence 


\section{NOTES}

1. Ce riche dossier de quinze articles rend comptedes travaux menés entre février 2017 et novembre 2018 dans le cadre du programme de recherche LégiPop, " projet structurant » financé par la MESHS Lille-Nord de France, coordonné par Anne Besson (Université d'Artois, «Textes et Cultures »). Trois événements scientifiques l'ont ponctué, le colloque « Des savants amateurs aux fans experts: contours et évolutions de l'érudition, entre populaire et académique " (16-17 novembre 2017, MESHS Lille-Nord de France, org. Anne Besson et Anne-Gaëlle Weber), les journées «(Dé)construire la légitimité » (16 mars 2018, MESHS Lille-Nord de France, org. Anne Besson, Matthieu Letourneux et Laura Muller-Thoma) et «Les nouvelles formes de la vulgarisation et de l'écriture du savoir » (15 juin 2018, Université Sorbonne Nouvelle - Paris 3, org. Anne Isabelle François et Sarah Lécossais). Nous reprenons donc en partie ici les textes des appels à contribution co-rédigés pour ces trois événements par les membres organisateurs de l'équipe LégiPop. Nous vous proposons ici une sélection d'articles tirés des communications données lors de ces trois rencontres. Nous remercions tous les participants à ces rencontres, y compris bien entendu ceux dont les communications n'ont pas donné lieu à des articles. Voir les programmes complets surle sitehttps://legipop.hypotheses.org/rencontres.

2. Les sens en sont en effet très fluctuants, historiquement par exemple : le "populaire " des années 1960 n'est pas plus le «populaire » des années 1980 qu'il n'est celui des années 2010. On tendra dans cette préface à considérer que le " populaire » (au sens neutre des cultural studies) est ce que les savants et ceux qui professent un savoir en dehors des institutions académiques entendent par «populaire» et que le «savant» est aussi ce que les «doctes » et ceux qui n'inscrivent pas leurs études dans le domaine des institutions scolaires ou académiques entendent par « savant " et par « légitime ».

3. Ils font l'objet des travaux de Julie Demange, qui a participé au colloque de novembre 2017. Sa thèse en cours enhistoire culturelle (dir. Pascal Ory) porte sur l'« Émergence et [la] construction du mouvement bédéphile en France dans les années 1960-1970».

4. Avec l'article «Le voleur de styles » paru dansL'Aiguille Preuve(revue de l'Association des Amis d'Arsène Lupin), n² 20, juin 2018.

5. Richard Peterson, «Understanding AudienceSegmentation. From Elite and Mass to Omnivore and Univore ", Poetics n² 21, 1992, p. 243-258.

6. Anne-Gaëlle Weber, Nicolas Wanlin, Evelyne Thoizet (dir.), Panthéons littéraires et savants (XIX ${ }^{\mathrm{e}}-\mathrm{XX}^{\mathrm{e}}$ siècles), Arras, Artois Presses Universités, 2012.Stéphane Zékian, L'Invention des classiques, Paris, CNRS Editions, 2012.

7. Diana Holmes, Middlebrow Matters. Women's reading and the literary canon in France since the Belle Époque, Liverpool University Press, 2018.

8. Guillaume Sibertin-Blanc,Legrand Stéphane, Esquisses d'une contribution à la critique de l'économie des savoirs, Reims, Le Clou dans le fer, 2009; Jack Stilgoe, Citizen Scientists: Reconnecting Science with Civil Society, London, Demos, 2009 ; Myriam Suchet, Indiscipline! Tentatives d'univercité à l'usage des littégraphistes, artistechniciens et autres philopraticiens, Montréal, Nota Bene, 2016.

9. Luc Boltanski, Pascale Maldidier, La vulgarisation scientifique et son public: enquête sur «Science et vie», Paris, Éd. de l'EHESS, 1977; Pierre Fayard, La communication scientifique publique: de la vulgarisation à la médiatisation, Lyon, Chronique sociale, 1988; Daniel Jacobi, Diffusion et vulgarisation : itinéraires du texte scientifique, Besançon, Annales littéraires de l'université de Besançon,PU Franche-Comté, 1986. 
INDEX

Mots-clés: savoir, culture, culture populaire, légitimité, expertise, fans, fan studies, savant, populaire, critique

\section{AUTHORS}

\section{ANNE BESSON}

Anne Besson est Professeure de Littérature Générale et Comparée à l'Université d'Artois(Arras). Spécialiste des ensembles romanesques et des constructions de mondes alternatifs, particulièrement en science-fiction, fantasy et littérature de jeunesse, elle est l'autrice deD'Asimov à Tolkien,cycles et séries dans la littérature de genre(CNRS Éditions, 2004), La Fantasy(Klincksieck, collection « 50 questions », 2007) etConstellations. Des mondes fictionnels dans l'imaginaire contemporain(CNRS Éditions,2015). Impliquée dans l'organisation et la diffusion des activités de recherche, co-fondatrice de l'association « Modernités médiévales ", elle a organisé de nombreux colloques, animé les sessions du MOOC « Fantasy » et du « MOOC SF », coordonné une douzaine d'ouvrages collectifs dont le récentDictionnaire de la Fantasy(Vendémiaire, 2018).

\section{ANNE ISABELLE FRANÇOIS}

Anne Isabelle François est maître de conférences de littérature comparée à l'Université Sorbonne Nouvelle - Paris 3. Spécialiste des domaines germanophone et anglophone des 20e et 21e siècles ainsi que des relations entre texte et image et de la culture populaire, elle poursuit ses recherches, dans une perspective deGenderet deCultural Studies, sur l'imaginaire occidental au sein du Centre d'Études et de Recherches Comparatistes (CERC - EA 172)

\section{SARAH LÉCOSSAIS}

Sarah Lécossais est maîtresse de conférences en sciences de l'information et de la communication à l'Université Paris 13, membre du LabSIC, et travaille sur les séries télévisées. Son travail de thèse portait sur les représentations de la parentalité au prisme du genre dans les séries télévisées françaises et elle s'intéresse désormais, toujours sous l'angle du genre, aux scénaristes en France. Elle a récemment codirigé un ouvrage avec Nelly Quemener sur les archives audiovisuelles intituléEn quête d'archives : bricolages méthodologiques en terrains médiatiquesaux éditions de l'INA (2018).

\section{MATTHIEU LETOURNEUX}

Matthieu Letourneux est Professeur à l'Université Paris Nanterre et membre du CSLF. Spécialiste des cultures sérielles et médiatiques, il a publiéFictions à la chaîne, Littératures sérielles et culture médiatique(Seuil, 2017),Cinéma, premiers crimes(avec A. Carou, Paris Bibliothèques ; 2015),Fantômas, Biographie d'un criminel imaginaire(avec L. Artiaga, Les Prairies Ordinaires, 2013),La Librairie Tallandier(avec J.-Y. Mollier, Nouveau Monde, 2011) etLe Roman d'aventures, 1870-1930(PULIM, 2010). Il a dirigé une dizaine de volumes collectifs et réédité les œuvres de Gustave Aimard, Gabriel Ferry, Emilio Salgari, Eugène Sue, Louis Forest et la sérieFantômas. Il estrédacteur en chef deBelphégor(http://belphegor.revues.org/). 


\section{ANNE-GAËLLE WEBER}

Anne-Gaëlle Weber est ancienne élève de l'ENS Ulm, agrégée de Lettres Modernes et professeur de littérature comparée à l'université d'Artois. Elle est spécialiste des rapports entre sciences et littératures en Occident aux XVIIIe et XIXe siècles. Elle est l'auteur, notamment, deLes Perroquets de Cook(Paris, Champion, 2013). 